\title{
Enhancement of Nucleoside Production in Hirsutella sinensis Based on Biosynthetic Pathway Analysis
}

\author{
Zhi-Qiang Liu, ${ }^{1}$ Bo Zhang, ${ }^{1}$ Shan Lin, ${ }^{1}$ Peter James Baker, ${ }^{1}$ \\ Mao-Sheng Chen, ${ }^{2}$ Ya-Ping Xue, ${ }^{1}$ Hui Wu, ${ }^{3}$ Feng Xu, ${ }^{3}$ Shui-Jin Yuan, ${ }^{3}$ Yi Teng, \\ Ling-Fang $\mathrm{Wu}^{3}{ }^{3}$ and $\mathrm{Yu}$-Guo Zheng ${ }^{1}$ \\ ${ }^{1}$ Key Laboratory of Bioorganic Synthesis of Zhejiang Province, College of Biotechnology and Bioengineering, \\ Zhejiang University of Technology, Hangzhou 310014, China \\ ${ }^{2}$ Department of Nephrology, Zhejiang Provincial People’s Hospital, Hangzhou 310014, China \\ ${ }^{3}$ East China Pharmaceutical Group Limited Co., Ltd, Hangzhou 311000, China \\ Correspondence should be addressed to Yu-Guo Zheng; zhengyg@zjut.edu.cn
}

Received 6 August 2017; Accepted 25 October 2017; Published 29 November 2017

Academic Editor: Zongbao K. Zhao

Copyright (c) 2017 Zhi-Qiang Liu et al. This is an open access article distributed under the Creative Commons Attribution License, which permits unrestricted use, distribution, and reproduction in any medium, provided the original work is properly cited.

\begin{abstract}
To enhance nucleoside production in Hirsutella sinensis, the biosynthetic pathways of purine and pyrimidine nucleosides were constructed and verified. The differential expression analysis showed that purine nucleoside phosphorylase, inosine monophosphate dehydrogenase, and guanosine monophosphate synthase genes involved in purine nucleotide biosynthesis were significantly upregulated 16.56-fold, 8-fold, and 5.43-fold, respectively. Moreover, dihydroorotate dehydrogenase, uridine nucleosidase, uridine/cytidine monophosphate kinase, and inosine triphosphate pyrophosphatase genes participating in pyrimidine nucleoside biosynthesis were upregulated 4.53-fold, 10.63-fold, 4.26-fold, and 5.98-fold, respectively. To enhance the nucleoside production, precursors for synthesis of nucleosides were added based on the analysis of biosynthetic pathways. Uridine and cytidine contents, respectively, reached $5.04 \mathrm{mg} / \mathrm{g}$ and $3.54 \mathrm{mg} / \mathrm{g}$ when adding $2 \mathrm{mg} / \mathrm{mL}$ of ribose, resulting in an increase of $28.6 \%$ and $296 \%$ compared with the control, respectively. Meanwhile, uridine and cytidine contents, respectively, reached $10.83 \mathrm{mg} / \mathrm{g} 2.12 \mathrm{mg} / \mathrm{g}$ when adding $0.3 \mathrm{mg} / \mathrm{mL}$ of uracil, leading to an increase of $176.3 \%$ and $137.1 \%$, respectively. This report indicated that fermentation regulation was an effective way to enhance the nucleoside production in $H$. sinensis based on biosynthetic pathway analysis.
\end{abstract}

\section{Introduction}

Ophiocordyceps sinensis (renamed from Cordyceps sinensis) has been well known and used as a traditional medicine or healthful food in China and is generally found on the Tibetan Plateau at high altitudes ranging from 3,600 to $5,400 \mathrm{~m}[1,2]$. Previous studies have revealed that $O$. sinensis and its anamorph possess a variety of biologically effective compounds with extensive pharmacological effects [3]. Due to the unique medicinal value, the natural $O$. sinensis is facing high demand and insufficient supply. It is reported that mycelia of the Cordyceps species produced by fermentation have become a feasible and sustainable means for producing the medicinal fungus and bioactive compounds [4]. Hirsutella sinensis is currently considered as the only correct anamorph of $O$. sinensis based on its morphological and phylogenetic characteristics [5]. Bioactive compounds from mycelia of $H$. sinensis by submerged cultivation have been reported to have similar clinical efficacy and less associated toxicity compared with wild $O$. sinensis, indicating that the submerged cultivation of $H$. sinensis has the trend of gradually replacing the $O$. sinensis in the market $[6,7]$. Therefore, with the increasing interest in Cordyceps both for the mycology and the pharmacology, it is more and more urgent to investigate the application of $H$. sinensis.

Nucleosides including purine and pyrimidine nucleosides are reported to possess many interesting forms of biological and pharmacological activity, such as anti-infective activity [8], antiviral activity [9], anticancer activity [10], antioxidation activity [11], and immunological stimulation [12], which implies a need for large-scale production of nucleosides. The concentration of nucleosides is also an 
important standard for quality control of $O$. sinensis. To date, more than 10 nucleosides, nucleobases, and their related compounds including adenine, adenosine, cytosine, cytidine, uridine, guanine, guanosine, hypoxanthine, vernine, thymidine, cordycepin, and $2^{\prime}$-deoxyuridine have been isolated from $O$. sinensis and identified [13]. Currently, three extraction methods, namely, organic solvent (methanol) pressurized liquid extraction (OSPLE), boiling water extraction (BWE), and ambient temperature water extraction (ATWE), are commonly applied for the extraction of nucleosides from natural and artificially cultured $O$. sinensis [14]. The effects of extraction methods on the quantification of nucleosides in different samples of $O$. sinensis are varied [15]. A rapid ultraperformance liquid chromatography method has been developed for the simultaneous determination of nucleoside from cultured Jiangxi O. sinensis, and cytosine (98.9 $\mu \mathrm{g} / \mathrm{g})$, uracil $(301.3 \mu \mathrm{g} / \mathrm{g})$, cytidine $(832.8 \mu \mathrm{g} / \mathrm{g})$, uridine $(3214.4 \mu \mathrm{g} / \mathrm{g})$, and adenine $(566.2 \mu \mathrm{g} / \mathrm{g})$ were determined [16]. Moreover, an ion-pairing reversed-phase liquid chromatography/mass spectrometry procedure was generated for the determination of nucleosides from $O$. sinensis, and the components of nucleosides including adenosine $(0.55-8.85 \mathrm{~g} / \mathrm{mL})$, uridine $(0.33-10.50 \mathrm{~g} / \mathrm{mL})$, cytidine $(0.48-15.30 \mathrm{~g} / \mathrm{mL})$, thymidine $(0.20-6.30 \mathrm{~g} / \mathrm{mL})$, guanine $(0.26-4.20 \mathrm{~g} / \mathrm{mL})$, uracil $(0.38-12.15 \mathrm{~g} / \mathrm{mL})$, cytosine $(0.39-12.45 \mathrm{~g} / \mathrm{mL})$, and thymine $(0.26-8.25 \mathrm{~g} / \mathrm{mL})$ were determined [17].

Previous studies have investigated the enhancement of the production of target products based on biosynthetic pathway analysis [18-20]. The cordycepin and cordycepic acid production from $H$. sinensis was improved based on biosynthetic pathway analysis and differential expression analysis [18]. The production of $n$-alkanes in Escherichia coli was significantly enhanced through spatial organization of enzymes involved in its biosynthetic pathway [19]. Metabolic engineering of the early non-mevalonate terpenoid pathway in $E$. coli was carried out to increase the supply of prenyl pyrophosphates as precursors for carotenoid production, and the carotenoid content reached $1.6 \mathrm{mg} / \mathrm{g}$ [20]. These studies indicated that it is feasible to enhance nucleoside production by analyzing and regulating their biosynthetic pathways. To date, there are few reports of enhancing the nucleoside production in $H$. sinensis. Our previous studies in transcriptome sequencing and analysis discovered the genes and enzymes involved in biosynthetic pathways, which benefited the investigation of biosynthetic pathway analysis for enhancing their production [21].

In this study, the biosynthetic pathways of purine and pyrimidine nucleosides in $H$. sinensis were constructed and verified. The differential expression analysis of the genes that encode the enzymes involved in the biosynthetic pathways for purine and pyrimidine nucleosides was performed, and the significantly upregulated genes corresponding to important enzymes were determined. Based on the analysis of biosynthetic pathways and gene differential expressions, fermentation regulation of $H$. sinensis was carried out, and the positive effects on nucleoside production were obtained. The fermentation regulation was observed to be efficient in enhancing the nucleoside production in $H$. sinensis.

\section{Materials and Methods}

2.1. Materials and Chemicals. H. sinensis L0106 was isolated from wild $O$. sinensis samples that were gingerly collected from Qinghai-Tibet Plateau in Qinghai Province. $H$. sinensis L0106 has been deposited at the China Center for Type Culture Collection (Wuhan, China) with accession number CCTCC M2011278. Subsequently, the transcriptome of $H$. sinensis was sequenced and the reads obtained were further assembled into Unigenes, and then BLASTx alignment $(e$ $<0.00001)$ between protein databases and Unigenes was performed [21]. E. coli JM109 was selected as host for plasmid pMD18-T transformation (Invitrogen, Carlsbad, CA, USA), and E. coli BL21 (DE3) (Invitrogen) was selected as the host for expression of pET28a (Invitrogen). Lysogeny broth (LB) medium (10 g/L tryptone, $5 \mathrm{~g} / \mathrm{L}$ yeast extract, and $10 \mathrm{~g} / \mathrm{L} \mathrm{NaCl}$ ) at $37^{\circ} \mathrm{C}$ with shaking (200 rpm) was used for the growth of E. coli transformants. Ampicillin, kanamycin, isopropyl- $\beta$-D-thiogalactopyranoside (IPTG), adenosine, vernine, cytidine, uridine, and thymidine standard substances were purchased from Sigma Chemical Co. (St. Louis, MO, USA).

2.2. Submerged Fermentation of $H$. sinensis. Slant agar medium for cultivation of $H$. sinensis consisted of powdered silkworm pupae (1.5\%), corn flour (1\%), bran (1.5\%), glucose $(1.5 \%)$, dextrin $(0.5 \%)$, yeast extract $(0.5 \%)$, tryptone $(1 \%)$, $\mathrm{MgSO}_{4}(0.01 \%), \mathrm{KH}_{2} \mathrm{PO}_{4}(0.02 \%)$, and agar (2\%). Powdered silkworm pupae, corn flour, and bran were first prepared and liquefied at $121^{\circ} \mathrm{C}$ for $20 \mathrm{~min}$, and then the liquefied medium obtained was used to dissolve other medium components. $H$. sinensis L0106 was cultured on solid slant medium at $16^{\circ} \mathrm{C}$ for approximately 30 days, and then the colonies with $2 \mathrm{~cm}$ diameter were formed and 5 of them were transferred to seed medium using an inoculating shovel at $16^{\circ} \mathrm{C}$ for 20 days on a rotary shaker at $150 \mathrm{rpm}$. Furthermore, $5 \%$ seed medium was inoculated with submerged fermentation medium in $500 \mathrm{~mL}$ shake flasks, and mycelia were asexually reproduced and harvested for approximately 10 days. Submerged fermentation medium and seed medium had the same components as slant medium without agar.

2.3. Screening and Verifying Biosynthetic Genes of Purine and Pyrimidine Nucleosides. To study the biosynthetic pathway of purine nucleotides in $H$. sinensis, the purine metabolism pathway (map00230) in $H$. sinensis was constructed based on the KEGG annotation of the $H$. sinensis transcriptome. Meanwhile, based on the pyrimidine metabolism pathway (map00240), which was involved in the KEGG metabolic pathway annotation of the $H$. sinensis transcriptome, the biosynthetic pathway of pyrimidine nucleosides in $H$. sinensis was constructed.

The functional genes and enzymes involved in the biosynthetic pathways of purine and pyrimidine nucleosides in $H$. sinensis were verified by gene cloning and protein expression [22-24]. PCR was performed to verify the genes encoding the enzymes involved in the biosynthetic pathways of purine and pyrimidine nucleosides. Primers were designed based on the predicted open reading frame (ORF) sequences of 
target genes (Tables S1 and S2, Supporting Information), and then the genes encoding the enzymes were subcloned to the pMD18-T vectors. Subsequently, the recombinant plasmids were successfully transformed to E. coli JM109 competent cells. The recombinant E. coli JM109 was cultivated in the shaker at $37^{\circ} \mathrm{C}$, and the recombinant plasmids were extracted using a plasmid extraction kit (Qiagen, Hilden, Germany). After being sequenced, the target genes were subcloned into pET28a vectors, and then the recombinant plasmids were further transferred to E. coli BL21 competent cells. After inducing by IPTG, the genes were expressed in E. coli BL21, and then the expressed proteins were detected by $12 \%$ sodium dodecyl sulfate polyacrylamide gel electrophoresis (SDSPAGE).

\subsection{Real-Time PCR. Three different cDNA libraries from} mycelia of $H$. sinensis cultured by submerged fermentation for 3 days (growth period), 6 days (before stable period), and 9 days (stable period) were prepared by a cDNA library construction kit (TaKaRa, Dalian, China) according to the manufacturer's protocols. Meanwhile, primers for real-time PCR were designed by the Primer Express tool (Applied Biosystems, Foster City, CA, USA), and the 18S rRNA gene was selected as the internal control. Real-time PCR mixture $(10 \mu \mathrm{L})$ was prepared and consisted of $1 \mu \mathrm{L}$ of cDNA from 3-day, 6-day, and 9-day samples, respectively, $5 \mu \mathrm{L}$ of SYBR Green PCR Master Mix (2x) (Promega), and $0.5 \mu \mathrm{L}$ $(100 \mu \mathrm{mol} / \mathrm{L})$ of each forward and reverse primer. Real-time PCR analyses were performed three times with independent RNA samples according to the temperature-time profile as follows: denaturation of $95^{\circ} \mathrm{C}$ for $2 \mathrm{~min}, 40$ cycles of $95^{\circ} \mathrm{C}$ for $15 \mathrm{sec}, 60^{\circ} \mathrm{C}$ for $1 \mathrm{~min}$.

\subsection{Differential Expression Analysis of Biosynthetic Genes.} The $2^{-\Delta \Delta \mathrm{Ct}}$ method was applied to calculate the relative expression levels by comparing the cycle thresholds (CTs) of the target genes with the CTs of the 18S rRNA gene. Differences in relative transcript expression levels of different samples were compared at the $p<0.05$ level among different culture periods of $H$. sinensis using Student's $t$-test. Eight primers of the gene-encoding enzymes involved in purine nucleotide biosynthesis (Table S3, Supporting Information), as well as thirteen primers of the gene-encoding enzymes involved in pyrimidine nucleoside biosynthesis (Table S4, Supporting Information), were used to amplify the target products. Differential expression of up- or downregulated genes detected in the microarray experiments was determined by real-time PCR. The significantly upregulated genes were determined and selected as important genes encoding important enzymes involved in biosynthetic pathways of purine and pyrimidine nucleosides in H. sinensis.

\subsection{Strategy of Fermentation Regulation Based on the Biosyn-} thetic Pathway. In the biosynthetic pathway of pyrimidine nucleosides, ribose and uracil are the two products catalyzed by uridine nucleosidase. Certain concentrations of ribose and uracil may cause product inhibition of uridine nucleosidase and lead to the accumulation of uridine. To investigate the effects of addition of uracil on production of pyrimidine nucleosides, $0,1.0,2.0,3.0$, and $4.0 \mathrm{mg} / \mathrm{mL}$ ribose or $0,0.3$, $0.6,1.2$, and $1.9 \mathrm{mg} / \mathrm{mL}$ uracil were added to the $100 \mathrm{~mL}$ of the submerged fermentation medium of $H$. sinensis according to preliminary experiments.

2.7. Determination of Nucleoside Content. Mycelia of $H$. sinensis were harvested after fermentation and washed three times with ultrapure water and then placed in an oven at $60^{\circ} \mathrm{C}$ for drying to constant weight. The dried mycelia were weighed, pounded to powder, and sealed under refrigeration at $4^{\circ} \mathrm{C}$ for further use. To analyze nucleoside content, $0.50 \mathrm{~g}$ of the dried powder of $H$. sinensis mycelia was accurately weighed and extracted with $10 \mathrm{~mL}$ of a solution of water and ethyl alcohol $(75: 25, \mathrm{v} / \mathrm{v})$, and then the mixture was placed in an ultrasonicator for $30 \mathrm{~min}$ and centrifuged at $12,000 \mathrm{rpm}$ for $10 \mathrm{~min}$. Supernatant was collected and the residue was reextracted twice. The extract obtained above was filtered through a $0.22 \mu \mathrm{m}$ filter and further analyzed. Assay of nucleoside content was carried out by high performance liquid chromatography (HPLC) according to a reported procedure with modifications [25]. The column temperature was maintained at $35^{\circ} \mathrm{C}$. The standards or samples were separated using a gradient mobile phase consisting of ultrapure water (A) and methyl alcohol (B). The gradient conditions are 0-3 min, 15\% B; 3.0-3.5 min, 15-25\% B; 3.5-8.55 min, 24\% B; 8.5-9.0 min, $24-35 \% \mathrm{~B}$; $9.0-15.0 \mathrm{~min}, 35 \% \mathrm{~B} ; 15.0-16.0 \mathrm{~min}$, $35-85 \% \mathrm{~B} ; 16.0-22.0 \mathrm{~min}, 85 \% \mathrm{~B} ; 22.0-22.5 \mathrm{~min}, 85-15 \% \mathrm{~B}$ and $22.5-27.5 \mathrm{~min}, 15 \% \mathrm{~B}$. The column was rinsed with $100 \%$ methyl alcohol for every ten runs. The flow rate was set at $1.0 \mathrm{~mL} / \mathrm{min}$. The peaks were detected at $260 \mathrm{~nm}$ and identified by comparing the retention times with the standard. A standard calibration curve was drawn by consecutively injecting different concentrations of standard adenosine, vernine, cytidine, uridine, and thymidine. The standard curves were prepared, and the linear regression equations are shown below: $Y_{1}=564.89 X_{1}-0.8929, R^{2}=0.9998$, where $Y_{1}$ stands for the adenosine concentration of the standard solution and $X_{1}$ stands for the peak area of adenosine; $Y_{2}$ $=477.01 X_{2}-1.3681, R^{2}=0.9951$, where $Y_{2}$ stands for the vernine concentration of the standard solution and $X_{2}$ stands for the peak area of vernine; $Y_{3}=321.19 X_{3}-0.2774, R^{2}$ $=0.9997$, where $Y_{3}$ stands for the cytidine concentration of the standard solution and $X_{3}$ stands for the peak area of cytidine; $Y_{4}=406.34 X_{4}+5.3884, R^{2}=0.9951$, where $Y_{4}$ stands for the uridine concentration of the standard solution and $X_{4}$ stands for the peak area of uridine; and $Y_{5}$ $=386.27 X_{5}-0.1233, R^{2}=0.9995$, where $Y_{5}$ stands for the thymidine concentration of the standard solution and $X_{5}$ stands for the peak area of thymidine. The percentage of nucleoside extraction yield (mg/g) was calculated as the nucleoside content of the extraction divided by dried sample weight.

2.8. Statistical Analysis. In this study, all experiments were performed in triplicate if not specifically noted. The data were analyzed by the statistical software SPSS (version 8.0, IBM, Chicago). Student's $t$-test and the analysis of variance (ANOVA) test were performed $(p<0.05)$. 


\section{Results}

3.1. Constructing Biosynthetic Pathways of Purine and Pyrimidine Nucleosides. The transcriptomes of $H$. sinensis in different cultivation periods ( 3 days, 6 days, and 9 days) were sequenced, the obtained reads were further assembled, and 20,822 Unigenes (All) were generated. For protein functional annotation, the Unigene sequences were searched using BLASTx against the protein databases [21]. From the analysis and annotation of $H$. sinensis transcriptome, most enzymes involved in biosynthetic pathways of purine and pyrimidine nucleosides were annotated (Tables S5 and S6, Supporting Information). Based on the annotated purine metabolic pathway (Figure S1, Supporting Information), the annotated enzymes were used to construct purine nucleotide biosynthesis, and a proposed biosynthetic pathway for purine nucleotides was obtained (Figure 1). In this biosynthetic pathway, purine nucleoside phosphorylase (punA) that converts adenosine to adenine, adenosine kinase that converts adenosine to adenosine $5^{\prime}$-monophosphate (AMP), adenine phosphoribosyltransferase (APRT) that converts adenine to AMP, AMP deaminase that converts AMP to IMP, inosine monophosphate (IMP) dehydrogenase that converts IMP to XMP, guanosine monophosphate (GMP) synthase that converts XMP to GMP, and guanine deaminase that converts GMP to guanine were, respectively, determined (Figure 1). Particularly, punA, adenosine kinase, APRT, AMP deaminase, IMP dehydrogenase, GMP synthase, and guanine deaminase that were involved in purine nucleotide biosynthesis were discovered in the annotation dataset of $H$. sinensis. Unigene14697_All was annotated to $A K$, Unigene1297_All was annotated to adenosine kinase, Unigene12674_All was annotated to APRT, Unigene13557_All and Unigene3652_All were annotated to AMP deaminase, Unigene6288_All and Unigene9455_All were annotated to IMP dehydrogenase, and Unigene11031_All, Unigene11950_All, and Unigene13696_All were annotated to GMP synthase. In addition, Unigene7330_All and Unigene9274_All were annotated to guanine deaminase.

Based on the annotated pyrimidine metabolic pathway (Figure S2, Supporting Information), the biosynthetic pathway of pyrimidine nucleosides in $H$. sinensis, which started from glutamine, was constructed (Figure 2). In this biosynthetic pathway, dihydroorotase that converts glutamine to dihydroorotate, dihydroorotate dehydrogenase that converts dihydroorotate to orotic acid, uridine monophosphate synthetase that converts orotic acid to UMP, 5 -nucleotidase that converts UMP to uridine, uridine nucleosidase that converts uridine to uracil, uridine kinase (udk) that converts cytidine to CMP, uridine/cytidine monophosphate (UMP-CMP) kinase that converts CMP to CDP, nucleoside-diphosphate kinase (ndk) that converts UDP to UTP, apyrase that converts CTP to CDP, inosine triphosphate pyrophosphatase (ITPA) that converts UMP to UTP, CTP synthase that converts UTP to CTP, and cytidine deaminase (cdd) that converts cytidine to uridine were annotated in the $H$. sinensis transcriptome. Specifically, Unigene8547_All, Unigene8750_All, Unigene11749_All, and Unigene8448_All were annotated to dihydroorotase,
Unigene13687_All and Unigene6100_All were annotated to dihydroorotate dehydrogenase, Unigene3048_All, Unigene3106_All, and Unigene3256_All were annotated to uridine monophosphate synthetase, Unigene10391_All and Unigene11624_All were annotated to $5^{\prime}$-nucleotidase, Unigene17827_All was annotated to uridine nucleosidase, Unigene7973_All was annotated to uridine kinase, Unigene11229_All and Unigene11252_All were annotated to UMP-CMP kinase, Unigene17829_All was annotated to nucleoside-diphosphate kinase (ndk), Unigene1156_All was annotated to apyrase, Unigene5342_All was annotated to ITPA, Unigene6007_All was annotated to CTP synthase, and, finally, Unigene11624_All was annotated to cytidine deaminase (cdd).

3.2. Verifying Biosynthetic Pathways of Purine and Pyrimidine Nucleosides. The genes involved in the biosynthetic pathways of purine and pyrimidine nucleotides were verified by PCR. One punA gene, one adenosine kinase gene, one APRT gene, two AMP deaminase genes, two IMP dehydrogenase genes, three GMP synthase genes, and two guanine deaminase genes were successfully cloned (Figure S3, Supporting Information), and the corresponding proteins expressed in E. coli BL21 were detected by SDSPAGE (Figure 3). In addition, four dihydroorotase genes, two dihydroorotate dehydrogenase genes, three uridine monophosphate synthetase genes, two $5^{\prime}$-nucleotidase genes, one uridine nucleosidase gene, one $u d k$ gene, two UMP-CMP kinase genes, one $n d k$ gene, one apyrase gene, one ITPA gene, one CTP synthase gene, and one $c d d$ gene were successfully cloned (Figure S4, Supporting Information). The corresponding proteins expressed in E. coli BL21 were detected by SDS-PAGE (Figure 4). Moreover, the gene and protein sequences of these enzymes have been deposited in GenBank (http://www.ncbi.nlm.nih.gov/WebSub/?tool=genbank), and the corresponding GenBank accession numbers are shown in Tables S5 and S6. These results indicated that the enzymes involved in biosynthetic pathways of purine and pyrimidine nucleosides could be produced or detected in $H$. sinensis.

3.3. The Differential Expression Analysis of Biosynthetic Genes. To detect the differential expression genes (DEGs) involved in the biosynthetic pathways of purine and pyrimidine nucleosides during different culture periods, an efficient procedure of real-time PCR assay with significant specificity and sensitivity was performed. For purine nucleotide biosynthesis, 7 upregulated and 1 downregulated genes were quantified by real-time PCR (Figure S5, Supporting Information). As shown in Figure 5, the relative expression level was upregulated 16.56-fold for purA gene, 8-fold for purE gene, 4.5fold for purF1 gene, and 5.43-fold for purF2 gene, when 9-day samples were compared with 3-day samples. For pyrimidine nucleoside biosynthesis, 10 upregulated and 3 downregulated genes were quantified by real-time PCR (Figure S6, Supporting Information). Meanwhile, the relative expression level of $p y n B$ gene was upregulated 4.54-fold, $p y n E$ gene was upregulated 10.63-fold, pynG gene was upregulated 4.26-fold, and pynJ gene was upregulated 5.98-fold, when 9-day samples were compared with 3-day samples (Figure 5). These results 


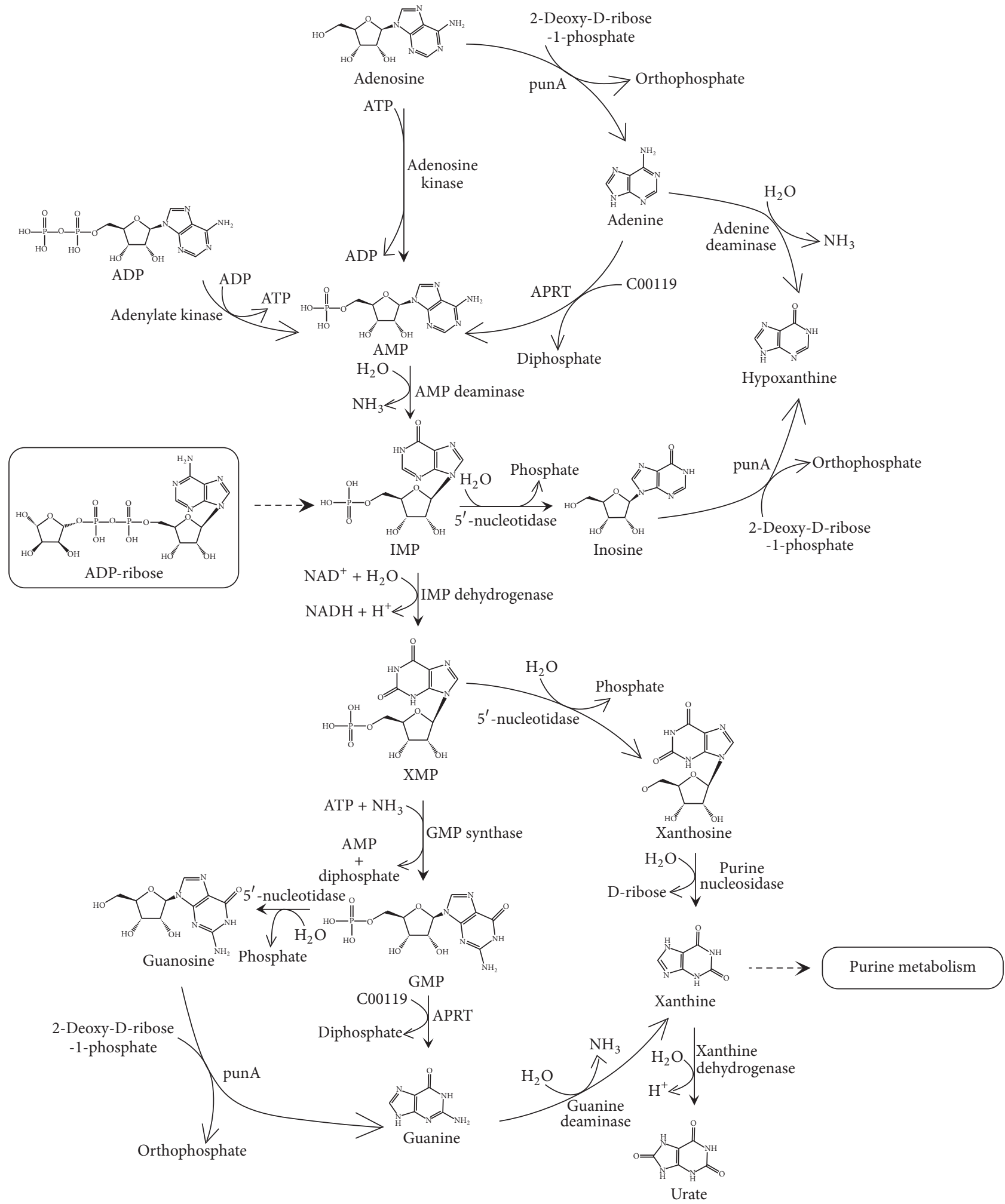

FIGURE 1: Biosynthetic pathway for purine nucleotides in $H$. sinensis. The biosynthetic pathway of purine nucleotides in $H$. sinensis was constructed based on KEGG annotation of $H$. sinensis transcriptome. The punA, adenosine kinase, APRT, AMP deaminase, IMP dehydrogenase, GMP synthase, and guanine deaminase involved in the biosynthetic pathway for purine nucleotides were annotated in the H. sinensis transcriptome. 


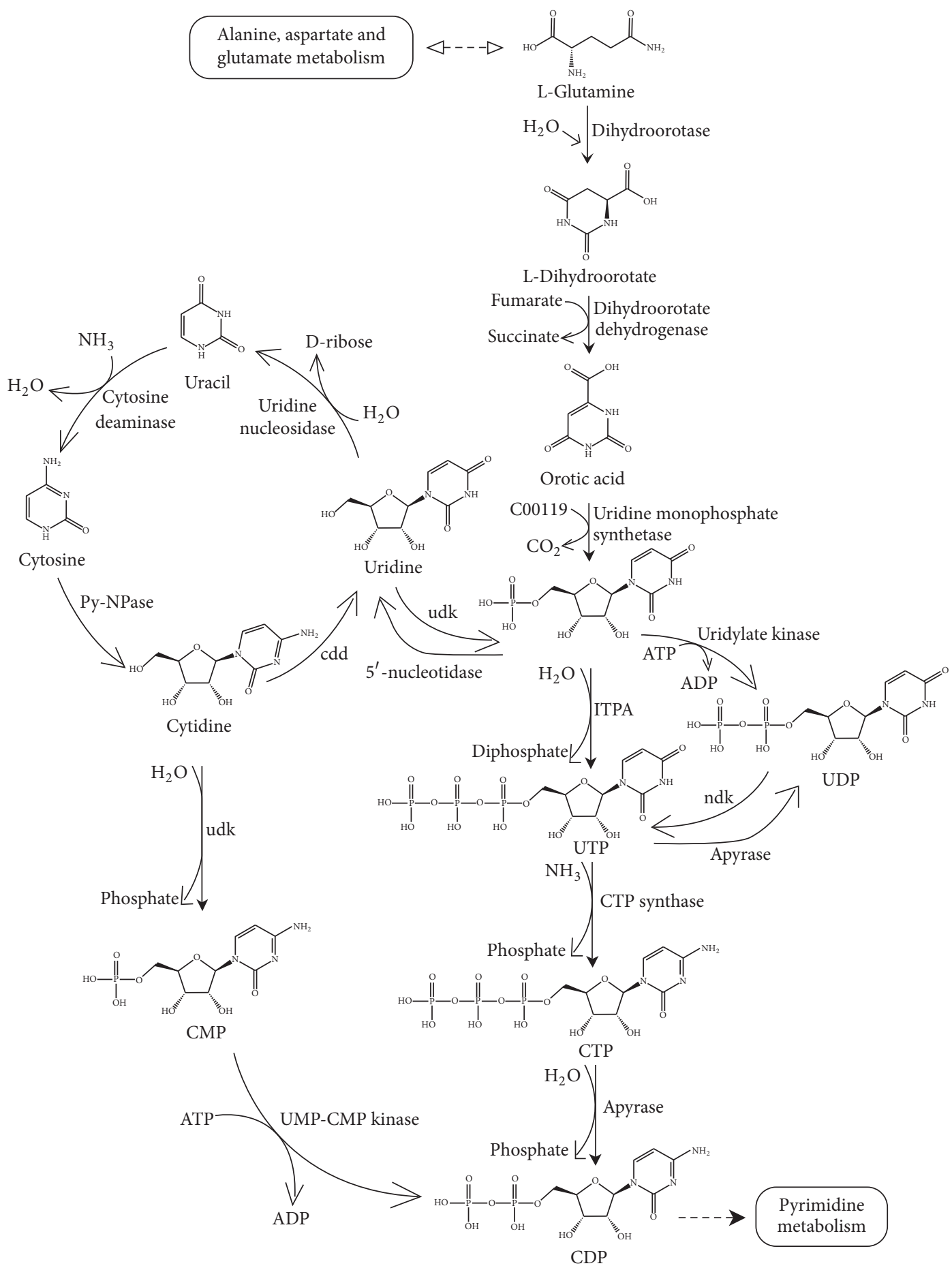

FIGURE 2: Biosynthetic pathway of pyrimidine nucleotides in H. sinensis. The biosynthetic pathway of pyrimidine nucleotides in $H$. sinensis was constructed based on KEGG annotation of the H. sinensis transcriptome. Dihydroorotase, dihydroorotate dehydrogenase, uridine monophosphate synthetase, $5^{\prime}$-nucleotidase, uridine nucleosidase, udk, UMP-CMP kinase, ndk, apyrase, ITPA, CTP synthase, and cdd involved in the biosynthetic pathway of pyrimidine nucleotides were annotated in the H. sinensis transcriptome.

indicated that punA, IMP dehydrogenase, and GMP synthase genes were overexpressed and played significant or stimulative roles in purine nucleotide biosynthesis. Dihydroorotate dehydrogenase, uridine nucleosidase, UMP-CMP kinase, and ITPA genes also played key roles in pyrimidine nucleoside biosynthesis.
3.4. Assay of Intracellular Nucleoside Production. The effects of different additives on nucleoside production were investigated by fermentation regulation in the submerged fermentation of $H$. sinensis. The results indicated that the outputs of adenosine, vernine, cytidine, uridine, and thymidine produced by $H$. sinensis were obviously enhanced compared 

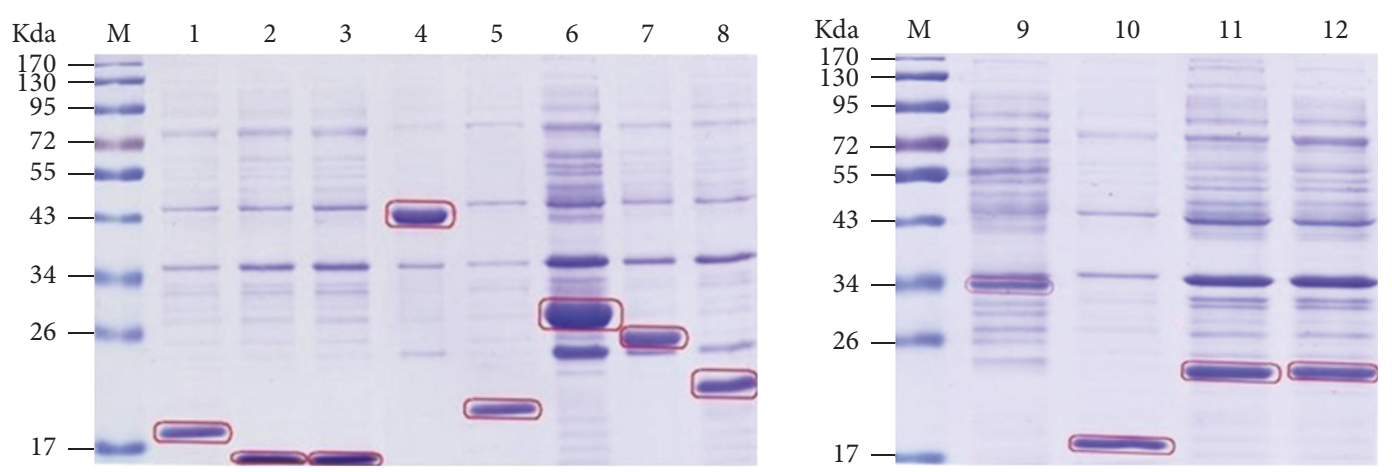

FiguRE 3: SDS-PAGE of expression products of biosynthetic genes of purine nucleotides in H. sinensis. Lane M, the protein marker, lane 1, purA, lane 2, purB, lane 3, purC, lane 4, purD2, lane 5, purE1, lane 6, purE2, lane 7, purF2, lane 8, purF3, lane 9, purG2, lane 10, purG1, lane 11, purD1, and lane 12, purF1.

with the control. For uridine production, the uridine content reached $5.04 \mathrm{mg} / \mathrm{g}$ with $28.6 \%$ increase over the control when the added concentration of ribose was $2 \mathrm{mg} / \mathrm{mL}$ (Figure 6), which was lower than the maximum content of uridine with $10.83 \mathrm{mg} / \mathrm{g}$ when the added concentration of uracil was $0.3 \mathrm{mg} / \mathrm{mL}$ (Figure 7), indicating that adding uracil was much better than ribose for uridine production. For adenosine production, the maximum content of adenosine reached $3.5 \mathrm{mg} / \mathrm{g}$ when the added concentration of ribose was $3 \mathrm{mg} / \mathrm{mL}$ (Figure 6), which was a $159.3 \%$ increase over the control and better than adenosine content with $2.11 \mathrm{mg} / \mathrm{g}$ when the added concentration of uracil was $0.3 \mathrm{mg} / \mathrm{mL}$ (Figure 7), indicating that adding ribose was better than uracil for adenosine production. For cytidine production, the maximum content of cytidine reached $3.54 \mathrm{mg} / \mathrm{g}$ when the added concentration of ribose was $2 \mathrm{mg} / \mathrm{mL}$ (Figure 6), which was a $296 \%$ increase over the control and better than the cytidine content of $2.12 \mathrm{mg} / \mathrm{g}$ when the added concentration of uracil was $0.6 \mathrm{mg} / \mathrm{mL}$ (Figure 7), indicating that adding ribose was better than uracil for cytidine production. In addition, for vernine and thymidine production, the maximum content of vernine reached $0.48 \mathrm{mg} / \mathrm{g}$ when the added concentration of ribose was $3 \mathrm{mg} / \mathrm{mL}$ (Figure 6), which was a 166.7\% increase over the control, and the maximum content of thymidine reached $0.87 \mathrm{mg} / \mathrm{g}$ when the added concentration of ribose was $2 \mathrm{mg} / \mathrm{mL}$ (Figure 6), indicating that the addition of ribose was more effective for the accumulation of thymidine compared with the addition of uracil.

\section{Discussion}

O. sinensis is well known to have been regarded as a traditional medicine or healthful food for a long time in China [26]. To meet the requirements of the market, submerged cultivation of $H$. sinensis provided an environmentally friendly way to resolve the demand $[27,28]$. In this study, nucleoside production in $H$. sinensis by submerged fermentation, especially after fermentation regulation, was satisfying or better compared with the nucleoside production reported in previous studies $[6,7]$, indicating that the submerged fermentation of $H$. sinensis had obvious advantages over culture of wild $O$. sinensis. Furthermore, most of the current studies focused on its medical applications, and there was little genetic information for $O$. sinensis [3]. The availability of transcriptome sequencing could lead to the identification and manipulation of candidate genes for the biosynthetic pathways of bioactive compounds.

As one of important bioactive compounds in H. sinensis, nucleoside content is a key factor controlling the quality of products in the submerged fermentation of $H$. sinensis. To date, there are few reports about investigating nucleoside production by submerged fermentation of $H$. sinensis, and the regulating biosynthetic pathways of the nucleosides in $H$. sinensis seem significant and important for enhancing the nucleoside production. Unfortunately, the biosynthetic pathway of the nucleosides has not been determined in $H$. sinensis. In plants, nucleotides could be synthesized from 5-phosphoribosyl-1-pyrophosphate and simple molecules or could be derived from preformed nucleosides and nucleobases via salvage reactions. Nucleotides are degraded to simple metabolites, and this process permits the recycling of phosphate, nitrogen, and carbon into central metabolic pools [29]. The biosynthesis of $3^{\prime}$-deoxyadenosine (cordycepin) in Cordyceps militaris has been investigated using [U$\left.{ }^{14} \mathrm{C}\right]$ adenosine and $\left[3-{ }^{3} \mathrm{H}\right]$ ribose, with the ${ }^{14} \mathrm{C}$ ratio in the adenine: ribose of the $\left[\mathrm{U}_{-}{ }^{14} \mathrm{C}\right]$ adenosine added to the $3^{\prime}$ deoxyadenosine producing cultures of $C$. militaris, which suggested that the formation of $2^{\prime}$-deoxynucleotide and $3^{\prime}$ deoxyadenosine may share a similar synthetic mechanism [30]. Zheng et al. carried out genome sequencing of $C$. militaris and constructed biosynthetic pathways for purine and adenosine based on the KEGG annotation, and it is found that the $5^{\prime}$-nucleotidase was an important enzyme involved in purine and adenosine biosynthesis [31]. The transcriptome of the medicinal $O$. sinensis fruiting body was analyzed by RNA_Seq; a proposed biosynthetic pathway for $3^{\prime}$-deoxyadenosine was constructed, which suggested that $5^{\prime}$-nucleotidase was an important enzyme involved in producing $3^{\prime}$-deoxyadenosine from $3^{\prime}$-dAMP. Adenosine kinase, adenylate kinase, and $5^{\prime}$-nucleotidase, which are involved in adenosine metabolic pathway and show the effects of phosphorylation and dephosphorylation, may also be involved in 

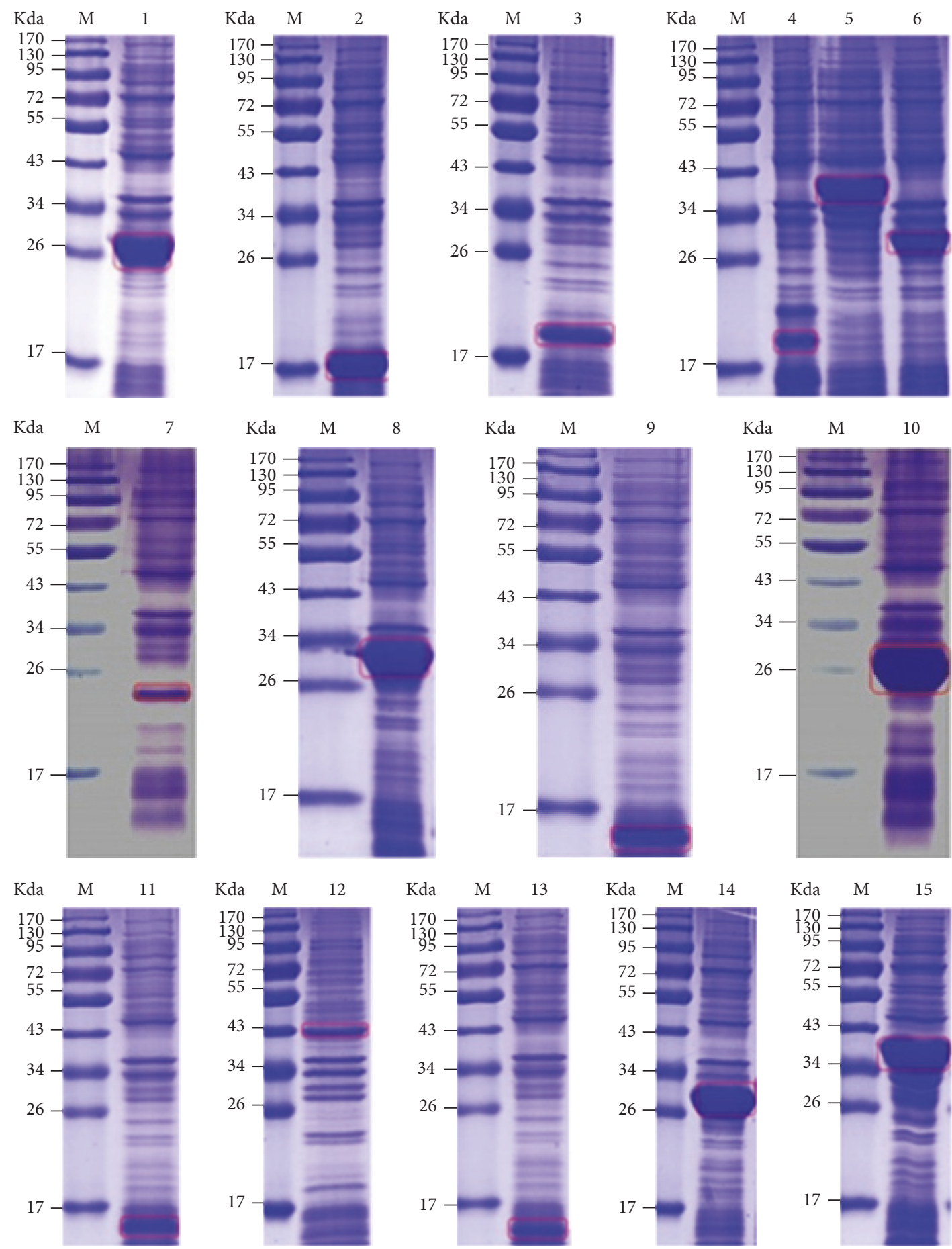

FIGURE 4: SDS-PAGE of expression products of biosynthetic genes of pyrimidine nucleotides in H. sinensis. Lane 1, pyrA3, lane 2, pyrB1, lane 3, pyrB2, lane 4, pyrC1, lane 5, pyrC2, lane 6, pyrC3, lane 7, pyrD1, lane 8, pyrE, lane 9, pyrF, lane 10, pyrG2, lane 11, pyrH, lane 12, pyrI, lane 13, pyrJ, lane 14, pyrK, and lane 15, pyrL.

the biosynthetic pathway of $3^{\prime}$-deoxyadenosine [32]. These previous studies provided evidences and references for the constructions of the biosynthetic pathways of nucleosides in $H$. sinensis, and the putative biosynthetic pathway had positive effects in guiding the enhancement of nucleoside production.
Differential expression analysis was frequently conducted to screen key genes in biosynthetic pathway, and real-time PCR has commonly been applied in relative expression level analysis for decades [33-35]. Gene annotation and differential expression analysis by real-time PCR identified 464 transcripts that may be involved in phytohormone catabolism 


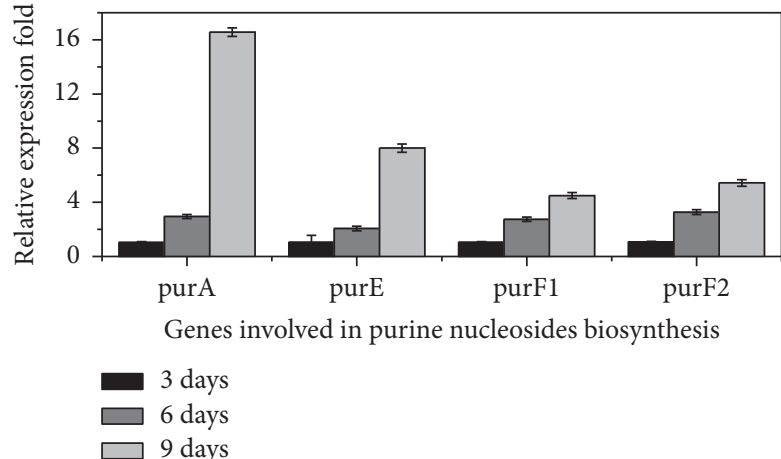

(a)

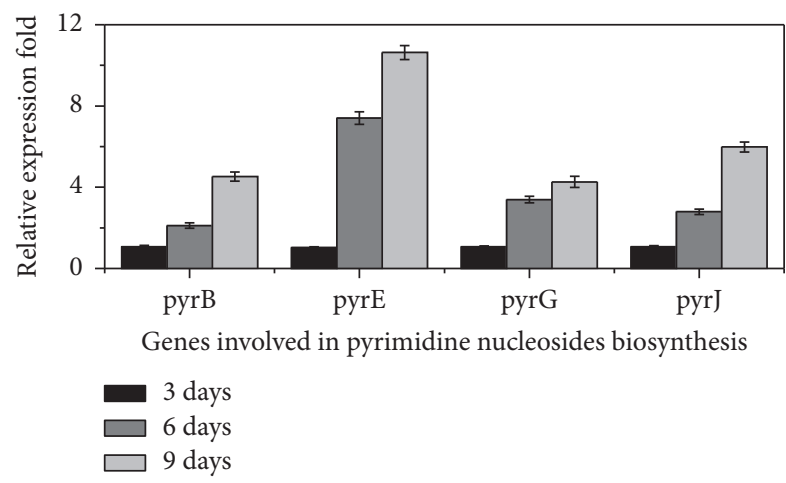

(b)

FIGURE 5: Significantly upregulated genes involved in biosynthetic pathways of purine and pyrimidine nucleotides in H. sinensis. (a) Upregulated genes involved in purine nucleotide biosynthesis. (b) Upregulated genes involved in pyrimidine nucleotide biosynthesis.

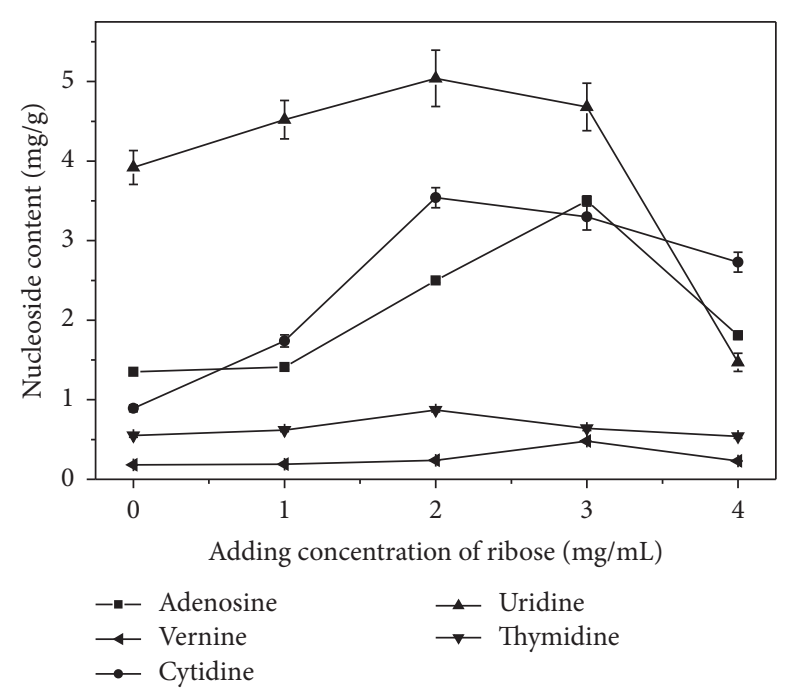

FigurE 6: The effects of adding different concentrations of ribose on nucleotide production in $H$. sinensis.

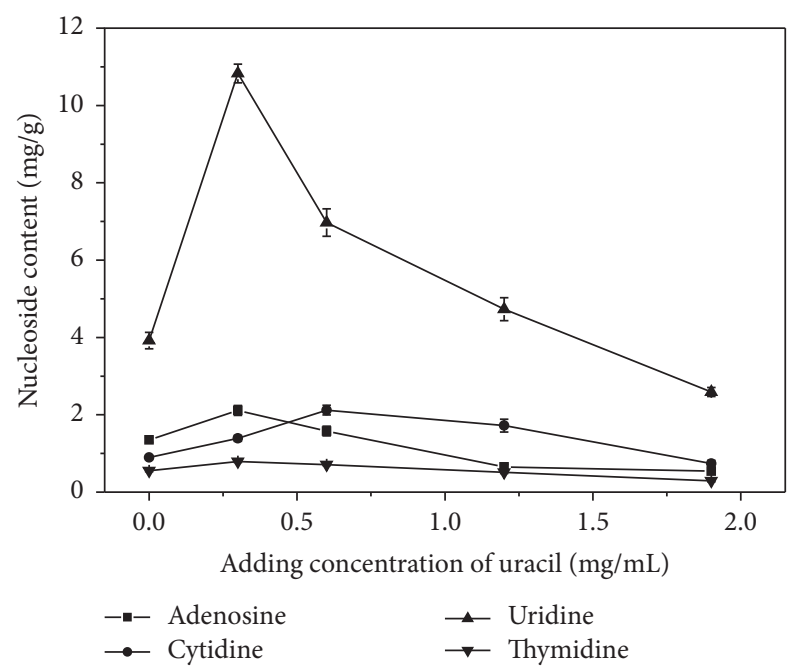

FIGURE 7: The effects of adding different concentrations of uracil on nucleotide production in $H$. sinensis. and biosynthesis, hormone signal, seed dormancy, seed maturation, cell wall growth, and circadian rhythms, particularly. Relative expression levels analysis showed that eleven phytohormone-related genes and five other genes have different expression patterns in the embryo and endosperm in the seed stratification process of Paris polyphylla [33]. Two proteases that are known to be directly involved in the process of pathogenesis in the entomopathogenic fungi Beauveria bassiana were identified through a comparative analysis of gene expression patterns and then verified by real-time PCR [34]. The differential expression of thirteen $\mathrm{PHB}$ accumulation-related genes was investigated by realtime PCR, and thirteen genes showed upregulated when the initial $\mathrm{C} / \mathrm{N}$ ratio was 2.4 , indicating that these genes played the important roles in $\mathrm{PHB}$ metabolism in Acidiphilium cryptum [35]. These previous studies suggested that realtime PCR is a reliable way to identify key genes involved in biosynthetic pathway. To our knowledge, there are few reports about differential expression analysis in $H$. sinensis, and no information about the regulation of biosynthetic pathways for production of secondary metabolites from $H$. sinensis. In this current study, significantly upregulated genes involved in the biosynthetic pathways of purine and pyrimidine nucleosides were identified by real-time PCR. Based on the results of the differential expression analysis, the uridine nucleosidase gene was significantly upregulated and uridine nucleosidase was considered as a key enzyme involved in the biosynthetic pathway of pyrimidine nucleosides. This study yielded more insight into the biosynthetic process for secondary metabolites and opened a significant way to determine key enzymes involved in a biosynthetic pathway.

Fermentation regulation was reported to enhance the production of target products with satisfying effects [20]. In this study, after fermentation regulation of $H$. sinensis based on biosynthetic pathway and differential expression analysis, the uridine production reached $5.04 \mathrm{mg} / \mathrm{g}$ with an increase of $28.6 \%$ when $2 \mathrm{mg} / \mathrm{mL}$ ribose was added, while uridine production reached $10.83 \mathrm{mg} / \mathrm{g}$ with a rise of $176.3 \%$ when $0.3 \mathrm{mg} / \mathrm{mL}$ uracil was added, indicating that the product inhibition for uridine nucleosidase by uracil was stronger 
than that by ribose. Some previous studies showed that ribose is an important precursor of nucleosides and uracil is usually considered as an intermediate in nucleoside biosynthesis [30, 36]. In this study, adenosine, cytidine, vernine, and thymidine productions by adding ribose were all better than those by adding uracil, which indicated that ribose played more important role than uracil in nucleoside biosynthesis. This study suggested that the effective fermentation regulation could be applied to improve the production of bioactive compounds and also demonstrated the availability of the constructed biosynthetic pathways.

\section{Conclusion}

In this study, biosynthetic pathways of purine and pyrimidine nucleosides were constructed and verified. Key genes were determined by real-time PCR, and punA, IMP dehydrogenase, and GMP synthase genes involved in purine nucleotide biosynthesis were found upregulated 16.56-fold, 8-fold, and 5.43-fold, respectively. In addition, dihydroorotate dehydrogenase, uridine nucleosidase, UMP-CMP kinase, and ITPA genes involved in pyrimidine nucleoside biosynthesis were found upregulated 4.53-fold, 10.63-fold, 4.26-fold, and 5.98fold, respectively. Regulation of $H$. sinensis fermentation was further conducted based on the biosynthetic pathways and gene differential expressions. Uridine, adenosine, and cytidine production, respectively, yielded an increase of $28.6 \%, 159.3 \%$, and $296 \%$ when ribose was added. In addition, uridine, adenosine, and cytidine production, respectively, had an increase of $176.3 \%, 56.3 \%$, and $137.1 \%$ when uracil was added.

\section{Conflicts of Interest}

No conflicts of interest exist.

\section{Acknowledgments}

The authors gratefully acknowledge the National High Technology Research and Development Program of China (no. 2012AA021701) and the Key Scientific and Technology Programs of Zhejiang Province (no. 2012C03005-1).

\section{Supplementary Materials}

Table S1. The primers used for cloning and expressing genes involved in biosynthetic pathway of purine nucleosides. Table S2. The primers used for cloning and expressing genes involved in biosynthetic pathway of pyrimidine nucleosides. Table S3. The primers involved in purine nucleosides biosynthesis used for real-time PCR. Table S4. The primers involved in pyrimidine nucleosides biosynthesis used for real-time PCR. Table S5. ORF analysis of functional genes involved in biosynthetic pathway of purine nucleotides in $H$. sinensis. Table S6. ORF analysis of functional genes involved in biosynthetic pathway of pyrimidine nucleotides in $H$. sinensis. Figure S1. The annotated purine metabolism pathway in $H$. sinensis. The red marks which stand for the corresponding enzymes were annotated in $H$. sinensis annotation dataset. Figure S2. The annotated pyrimidine metabolism pathway in $H$. sinensis. The red marks which stand for the corresponding enzymes were annotated in $H$. sinensis annotation dataset. Figure S3. Agarose gel electrophoresis of PCR products in biosynthetic pathway of purine nucleotides. Line A stands for purA, lane B1 stands for purB, lane C stands for purC, lane D1 stands for purD1, lane D2 stands for purD2, lane E1 stands for purE1, lane E2 stands for purE2, lane F1 stands for purF1, lane F2 stands for purF2, lane F3 stands for purF3, lane G1 stands for purG1, and lane G2 stands for purG2. Figure S4. Agarose gel electrophoresis of PCR products in biosynthetic pathway of pyrimidine nucleotides. Lane A1 stands for pyrA1, lane A2 stands for pyrA2, lane A3 stands for pyrA3, lane A4 stands for pyrA4, lane B1 stands for pyrB1, lane B2 stands for pyrB2, lane $\mathrm{C} 1$ stands for pyrC1, lane $\mathrm{C} 2$ stands for pyrC2, lane C3 stands for pyrC3, lane D1 stands for pyrD1, lane D2 stands for pyrD2, lane E stands for pyrE, lane F stands for pyrF, lane G1 stands for pyrG1, lane G2 stands for pyrG2, lane $\mathrm{H}$ stands for pyrH, lane I stands for pyrI, lane J stands for pyrJ, lane $\mathrm{K}$ stands for pyrK, and lane L stands for pyrL. Figure S5. Differential expression analysis of other four functional genes involved in biosynthetic pathway of purine nucleotides. Figure S6. Differential expression analysis of other nine functional genes involved in biosynthetic pathway of pyrimidine nucleosides. (Supplementary Materials)

\section{References}

[1] D. Winkler, "Yartsa Gunbu (Cordyceps sinensis) and the fungal commodification of Tibet's rural economy," Economic Botany, vol. 62, no. 3, pp. 291-305, 2008.

[2] C.-H. Dong and Y.-J. Yao, "Nutritional requirements of mycelial growth of Cordyceps sinensis in submerged culture," Journal of Applied Microbiology, vol. 99, no. 3, pp. 483-492, 2005.

[3] M. Singh, R. Tulsawani, P. Koganti, A. Chauhan, M. Manickam, and K. Misra, "Cordyceps sinensis increases hypoxia tolerance by inducing heme oxygenase-1 and metallothionein via Nrf2 activation in human lung epithelial cells," BioMed Research International, vol. 2013, Article ID 569206, 13 pages, 2013.

[4] Y. Liu, P. Leung, and J. Wu, "Exopolysaccharide production in batch and semi-continuous fermentation of Cordyceps sinensis," Journal of Biotechnology, vol. 136, pp. S301-S302, 2008.

[5] Z.-Y. Liu, Y.-J. Yao, A.-Y. Liu, D. N. Pegler, and M. W. Chase, "Molecular evidence for the anamorph-teleomorph connection in Cordyceps sinensis," Mycological Research, vol. 105, no. 7, pp. 827-832, 2001.

[6] S. Yu, Y. Zhang, and M. Fan, "Analysis of volatile compounds of mycelia of Hirsutella sinensis, the anamorph of ophiocordyceps sinensis," Applied Mechanics and Materials, vol. 140, no. 2, pp. 253-257, 2012.

[7] S. Lin, Z.-Q. Liu, P. J. Baker et al., "Enhancement of cordyceps polysaccharide production via biosynthetic pathway analysis in Hirsutella sinensis," International Journal of Biological Macromolecules, vol. 92, pp. 872-880, 2016.

[8] X. Zhou, C. U. Meyer, P. Schmidtke, and F. Zepp, "Effect of cordycepin on interleukin-10 production of human peripheral blood mononuclear cells," European Journal of Pharmacology, vol. 453, no. 2-3, pp. 309-317, 2002. 
[9] K. Nakamura, K. Konoha, N. Yoshikawa et al., "Effect of cordycepin (3/-deoxyadenosine) on hematogenic lung metastatic model mice," In Vivo, vol. 19, no. 1, pp. 137-142, 2005.

[10] J. V. De Julián-Ortiz, J. Gálvez, C. Muñoz-Collado, R. GarcíaDomenech, and C. Gimeno-Cardona, "Virtual combinatorial syntheses and computational screening of new potential antiherpes compounds," Journal of Medicinal Chemistry, vol. 42, no. 17, pp. 3308-3314, 1999.

[11] Y.-J. Tsai, L.-C. Lin, and T.-H. Tsai, "Pharmacokinetics of adenosine and cordycepin, a bioactive constituent of Cordyceps sinensis in rat," Journal of Agricultural and Food Chemistry, vol. 58, no. 8, pp. 4638-4643, 2010.

[12] H.-P. Wei, X.-L. Ye, Z. Chen et al., "Synthesis and pharmacokinetic evaluation of novel $\mathrm{N}$-acyl-cordycepin derivatives with a normal alkyl chain," European Journal of Medicinal Chemistry, vol. 44, no. 2, pp. 665-669, 2009.

[13] S. P. Li, F. Q. Yang, and K. W. K. Tsim, "Quality control of Cordyceps sinensis, a valued traditional Chinese medicine," Journal of Pharmaceutical and Biomedical Analysis, vol. 41, no. 5, pp. 1571-1584, 2006.

[14] F. Q. Yang and S. P. Li, "Effects of sample preparation methods on the quantification of nucleosides in natural and cultured Cordyceps," Journal of Pharmaceutical and Biomedical Analysis, vol. 48, no. 1, pp. 231-235, 2008.

[15] F.-Q. Guo, A. Li, L.-F. Huang, Y.-Z. Liang, and B.-M. Chen, "Identification and determination of nucleosides in Cordyceps sinensis and its substitutes by high performance liquid chromatography with mass spectrometric detection," Journal of Pharmaceutical and Biomedical Analysis, vol. 40, no. 3, pp. 623630, 2006.

[16] F. Q. Yang, J. Guan, and S. P. Li, "Fast simultaneous determination of 14 nucleosides and nucleobases in cultured Cordyceps using ultra-performance liquid chromatography," Talanta, vol. 73, no. 2, pp. 269-273, 2007.

[17] F. Q. Yang, D. Q. Li, K. Feng, D. J. Hu, and S. P. Li, "Determination of nucleotides, nucleosides and their transformation products in Cordyceps by ion-pairing reversed-phase liquid chromatography-mass spectrometry," Journal of Chromatography A, vol. 1217, no. 34, pp. 5501-5510, 2010.

[18] S. Lin, Z.-Q. Liu, Y.-P. Xue et al., "Biosynthetic Pathway Analysis for Improving the Cordycepin and Cordycepic Acid Production in Hirsutella sinensis," Applied Biochemistry and Biotechnology, vol. 179, no. 4, pp. 633-649, 2016.

[19] Z. Rahman, B. H. Sung, J.-Y. Yi, L. M. Bui, J. H. Lee, and S. C. Kim, "Enhanced production of n-alkanes in escherichia coli by spatial organization of biosynthetic pathway enzymes," Journal of Biotechnology, pp. 187-191, 2014.

[20] M. Albrecht, N. Misawa, and G. Sandmann, "Metabolic engineering of the terpenoid biosynthetic pathway of Escherichia coli for production of the carotenoids $\beta$-carotene and zeaxanthin," Biotechnology Letters, vol. 21, no. 9, pp. 791-795, 1999.

[21] Z.-Q. Liu, S. Lin, J. Baker et al., "Transcriptome sequencing and analysis of the entomopathogenic fungus Hirsutella sinensis isolated from Ophiocordyceps sinensis," BMC Genomics, vol. 16, no. 1 , article no. 106, 2015.

[22] J. Reboul, P. Vaglio, J.-F. Rual et al., "C. elegans ORFeome version 1.1: Experimental verification of the genome annotation and resource for proteomescale protein expression," Nature Genetics, vol. 34, no. 1, pp. 35-41, 2003.

[23] Z.-Q. Liu, J.-J. Ye, Z.-Y. Shen et al., "Upscale production of ethyl (S)-4-chloro-3-hydroxybutanoate by using carbonyl reductase coupled with glucose dehydrogenase in aqueous-organic solvent system," Applied Microbiology and Biotechnology, vol. 99, no. 5, pp. 2119-2129, 2015.

[24] Z.-Q. Liu, L.-M. Zhou, P. Liu et al., "Efficient two-step chemoenzymatic synthesis of all-trans-retinyl palmitate with high substrate concentration and product yield," Applied Microbiology and Biotechnology, vol. 99, no. 21, pp. 8891-8902, 2015.

[25] L. Deng, T.-Y. Zhou, L. Pi et al., "Optimization of microwaveassisted extraction of cordycepic acid and cordycepin from cultured cordyceps militaris by response surface methodology," Asian Journal of Chemistry, vol. 25, no. 14, pp. 8065-8071, 2013.

[26] R. Stone, "Last stand for the body snatcher of the Himalayas?" Science, vol. 322, no. 5905, p. 1182, 2008.

[27] J. C. Holliday and M. Cleaver, "Medicinal value of the caterpillar fungi species of the genus Cordyceps (Fr.) link (Ascomycetes). A review," International Journal of Medicinal Mushrooms, vol. 10, no. 3, pp. 219-234, 2008.

[28] R. Stone, "Improbable partners aim to bring biotechnology to a himalayan kingdom," Science, vol. 327, no. 5968, pp. 940-941, 2010.

[29] R. Zrenner, M. Stitt, U. Sonnewald, and R. Boldt, "Pyrimidine and purine biosynthesis and degradation in plants," Annual Review of Plant Biology, vol. 57, pp. 805-836, 2006.

[30] M. B. Lennon and R. J. Suhadolnik, "Biosynthesis of 3/deoxyadenosine by Cordyceps militaris. Mechanism of reduction," BBA Section Nucleic Acids And Protein Synthesis, vol. 425, no. 4, pp. 532-536, 1976.

[31] P. Zheng, Y. Xia, G. Xiao et al., "Genome sequence of the insect pathogenic fungus Cordyceps militaris, a valued traditional chinese medicine," Genome Biology, vol. 12, no. 11, article no. R116, 2011.

[32] L. Xiang, Y. Li, Y. Zhu et al., "Transcriptome analysis of the Ophiocordyceps sinensis fruiting body reveals putative genes involved in fruiting body development and cordycepin biosynthesis," Genomics, vol. 103, no. 1, pp. 154-159, 2014.

[33] J. Qi, N. Zheng, B. Zhang et al., "Mining genes involved in the stratification of Paris Polyphylla seeds using high-throughput embryo Transcriptome sequencing," BMC Genomics, vol. 14, no. 1, article no. 358, 2013.

[34] A. A. K. Pathan, K. Uma Devi, H. Vogel, and A. Reineke, "Analysis of differential gene expression in the generalist entomopathogenic fungus Beauveria bassiana (Bals.) Vuillemin grown on different insect cuticular extracts and synthetic medium through cDNA-AFLPs," Fungal Genetics and Biology, vol. 44, no. 12, pp. 1231-1241, 2007.

[35] A.-L. Xu, J.-L. Xia, K.-K. Liu et al., "Real-time PCR analysis of metabolic pathway of PHB in Acidiphilium cryptum DX1-1," Journal of Microbiology and Biotechnology, vol. 20, no. 1, pp. 7177, 2010.

[36] K. A. Jacobson, R. G. Ravi, E. Nandanan et al., "Ribose modified nucleosides and nucleotides as ligands for purine receptors," Nucleosides, Nucleotides and Nucleic Acids, vol. 20, no. 4-7, pp. 333-341, 2001. 

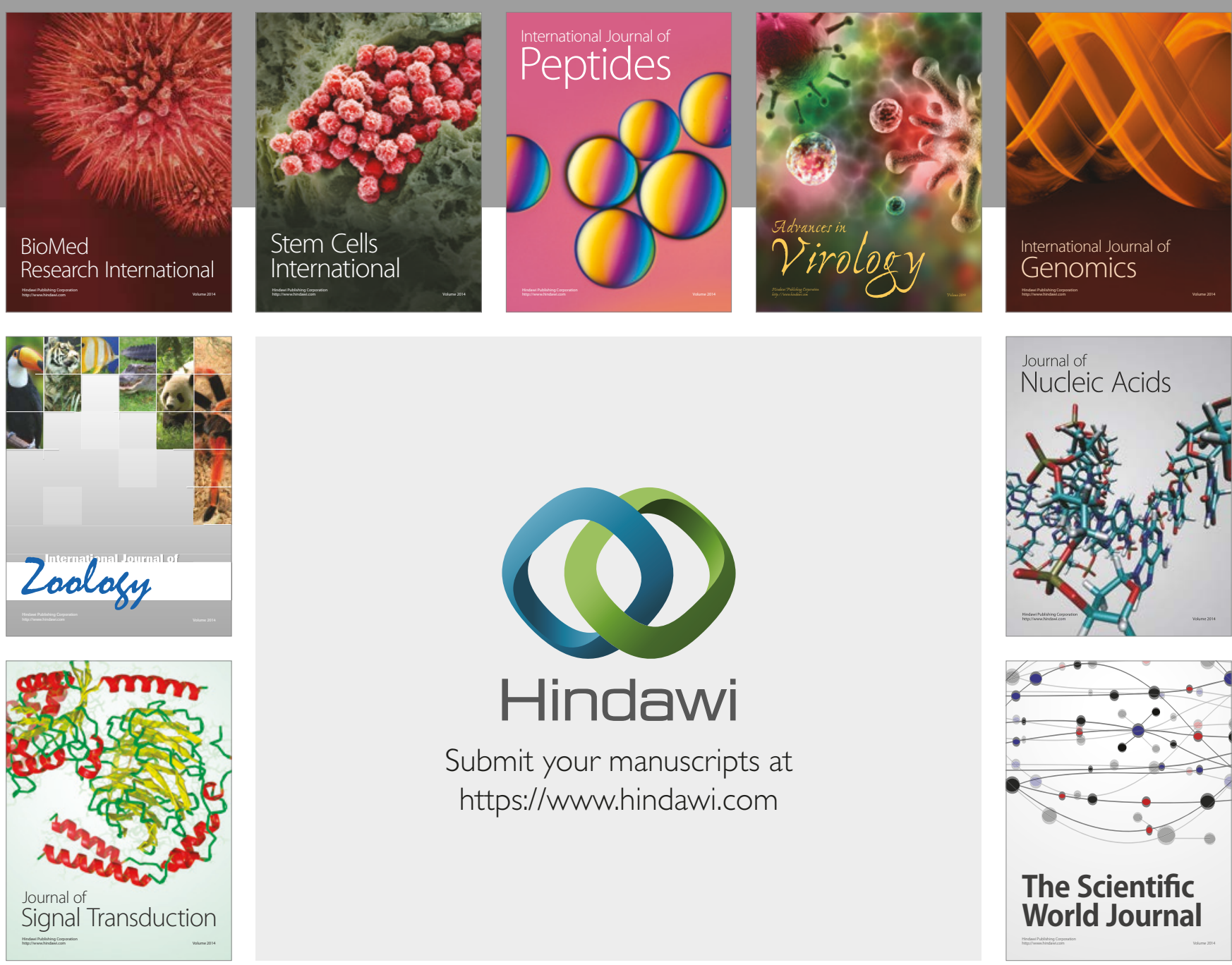

Submit your manuscripts at

https://www.hindawi.com
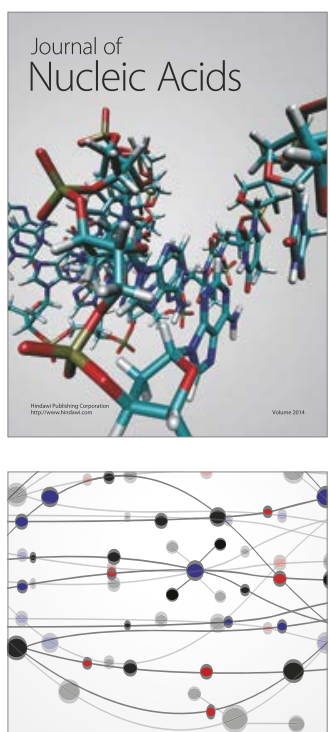

The Scientific World Journal

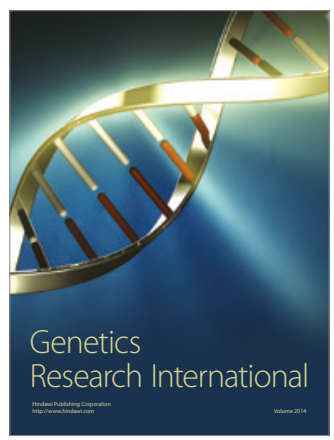

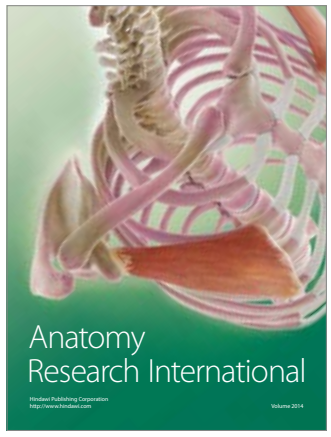

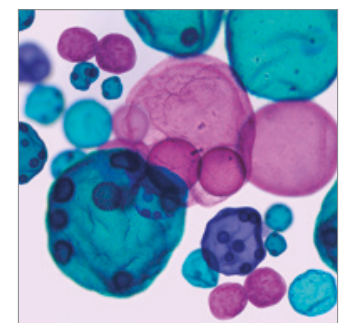

International Journal of Microbiology
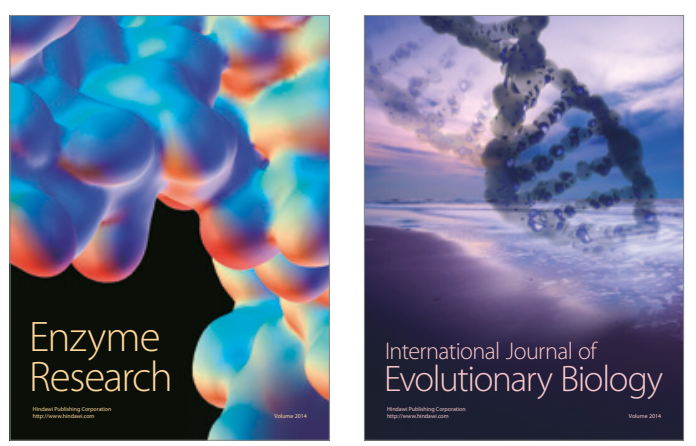
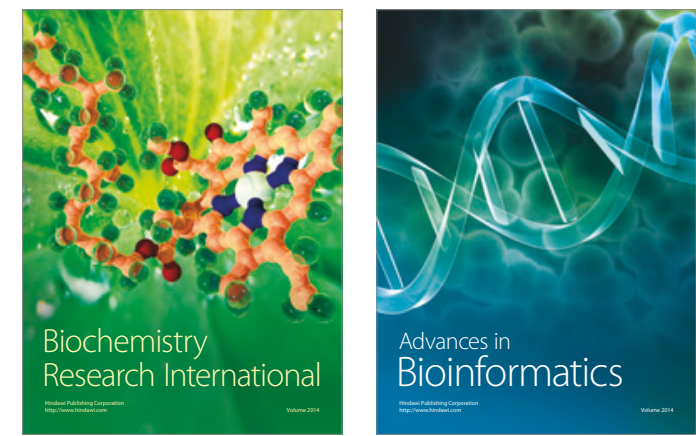

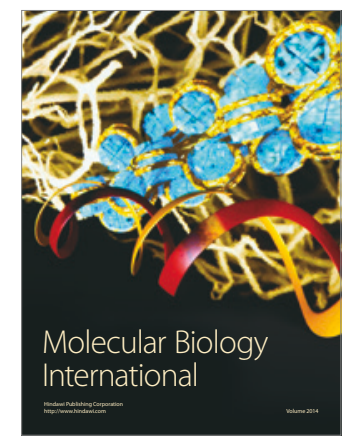

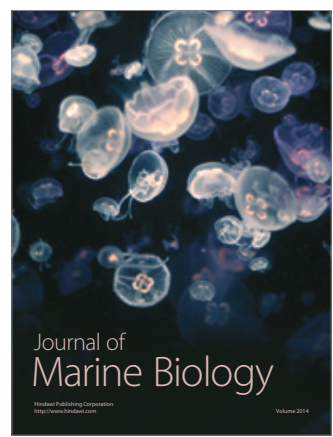

